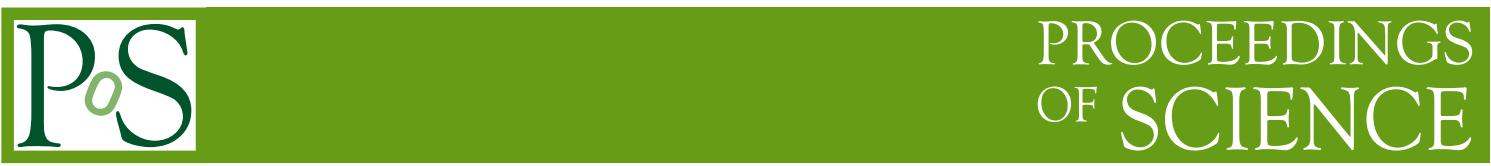

\title{
Charm baryons at LHCb
}

\author{
Dana Bobulska ${ }^{a, 1, *}$ \\ ${ }^{a}$ University of Glasgow, \\ Glasgow, United Kingdom \\ E-mail: dana.bobulska@cern.ch
}

The LHCb experiment collected the world's largest sample of charmed hadrons during LHC Run 1 and Run 2. With this data set, $\mathrm{LHCb}$ is currently providing the world's most precise measurements of properties and production of known charmed baryons, as well as searching for many previously unobserved states. The latest results on the charmed baryons described in this document include the lifetime measurement of the $\Omega_{c}^{0}$ and $\Xi_{c}^{0}$ baryons that are produced directly from proton-proton collisions and reconstructed in the $p K^{-} K^{-} \pi^{+}$final state. The $\Omega_{c}^{0}$ lifetime is measured to be $276.5 \pm 13.4$ (stat) \pm 4.4 (syst) $\pm 0.7\left(D^{0}\right)$ fs and the $\Xi_{c}^{0}$ lifetime to be $148.0 \pm 2.3$ (stat) \pm 2.2 (syst) $\pm 0.2\left(D^{0}\right)$ fs. Both results are consistent with the previous LHCb measurement based on semileptonic decays of $b$-hadrons. The latest results on the charm baryons from the LHCb collaboration also include searches for the $\Xi_{c c}^{+}$baryon in the $\Xi_{c c}^{+} \rightarrow \Xi_{c}^{+} \pi^{-} \pi^{+}$ decay, the first search for the $\Omega_{c c}^{+}$baryon in the $\Omega_{c c}^{+} \rightarrow \Xi_{c}^{+} K^{-} \pi^{+}$decay, and the searches for the $\Xi_{b c}^{0}$ and $\Omega_{b c}^{0}$ baryons in the $\Lambda_{c}^{+} \pi^{-}$and $\Xi_{c}^{+} \pi^{-}$final states. No significant signals are found for neither of these decays, therefore upper limits are set on the ratio of production cross-sections times the ratio of branching fractions with respect to their normalisation decay.

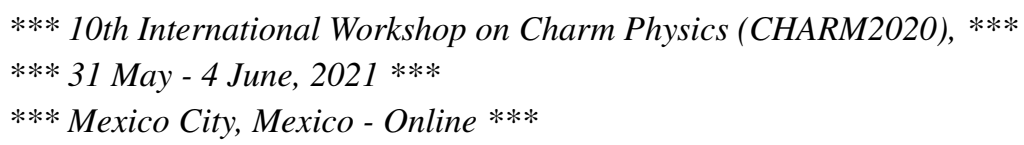




\section{Introduction}

In the last decade, 62 new hadrons were observed at the Large Hadron Collider (LHC), 55 of which were discovered by the LHCb experiment. Different properties of these hadrons, together with many other previously observed states, have been precisely studied by the LHCb experiment. Measurement of heavy-flavour hadron properties, such as their masses, lifetimes, and branching fractions, is of great importance as it is a valuable input for testing quantum chromodynamics predictions and to provide a deeper understanding of the hadronic structure. This document presents a measurement of lifetimes of the $\Omega_{c}^{0}$ and $\Xi_{c}^{0}$ baryons in Sec. 2; two searches for doubly charmed baryons $\Xi_{c c}^{+}$and $\Omega_{c c}^{+}$in Sec. 3; and searches for beauty-charm baryons $\Xi_{b c}^{0}$ and $\Omega_{b c}^{0}$ in Sec. 4; before concluding in Sec. 5 .

\section{Charmed baryon lifetimes}

\subsection{Introduction}

Theory predictions for the lifetime hierarchy of the charmed baryons are based on heavy quark expansion theory [1]. The lowest-order term in the expansion is calculated in inverse powers of the mass of the heavy quark, however also higher-order contributions, such as Pauli interference and weak $W$-boson annihilation, are needed for the charmed hadrons [2]. The lifetime hierarchy is predicted to be either $\tau\left(\Xi_{c}^{+}\right)>\tau\left(\Lambda_{c}^{+}\right)>\tau\left(\Xi_{c}^{0}\right)>\tau\left(\Omega_{c}^{0}\right)$ or $\tau\left(\Xi_{c}^{+}\right)>\tau\left(\Omega_{c}^{0}\right)>\tau\left(\Lambda_{c}^{+}\right)>$ $\tau\left(\Xi_{c}^{0}\right)$, depending on the treatment of those higher-order effects. Recently, the LHCb collaboration performed a measurement of charmed baryon lifetimes using semileptonic $b$-hadron decays [3, 4], where the lifetime of the $\Omega_{c}^{0}$ was measured to be $268 \pm 24$ (stat) \pm 10 (syst) $\pm 2\left(D^{+}\right.$) fs, almost four times larger than the world average [5] and inconsistent with it at the level of 7 standard Gaussian deviations $(\sigma)$, and the lifetime of the $\Xi_{c}^{0}$ baryon was measured to be $54.5 \pm 1.7$ (stat) \pm 1.6 (syst) \pm $1.0\left(D^{+}\right)$fs and in tension with the world average [5] at the level of $3.3 \sigma$. Additional measurements to confirm or rule out these results and to establish the lifetime hierarchy of the charmed baryons from the experimental side are therefore essential.

\subsection{Measurement}

The new measurement of the lifetimes of the $\Omega_{c}^{0}$ and $\Xi_{c}^{0}$ baryons uses baryons produced at the primary proton-proton $(p p)$ collision vertex $(\mathrm{PV})$, referred to as prompt decays, collected with the LHCb detector at a centre-of-mass energy of $13 \mathrm{TeV}$, corresponding to a total integrated luminosity of $5.4 \mathrm{fb}^{-1}$ [6]. Both baryons are reconstructed in the $p K^{-} K^{+} \pi^{+}$final state. This measurement is statistically independent of the previous LHCb measurement, and since it uses a different method to measure the baryon lifetimes, most of the systematic uncertainties are uncorrelated. In order to avoid experimenter's bias, the measured values for the lifetimes were not examined until the full procedure had been finalised. The lifetimes are measured relative to the $D^{0}$ lifetime using prompt $D^{0} \rightarrow K^{-} K^{+} \pi^{-} \pi^{+}$decays. Prompt signal yields are extracted with $2 \mathrm{D}$ extended maximum likelihood fits to the invariant mass $(m)$ and logarithm of $\chi_{\mathrm{IP}}^{2}$, where $\chi_{\mathrm{IP}}^{2}$ is defined as the difference in the vertex-fit $\chi^{2}$ of a given PV reconstructed with and without the track under consideration. The invariant mass is used to discriminate between signal and combinatorial background, whereas the $\log _{10} \chi_{\mathrm{IP}}^{2}$ variable is useful to distinguish between prompt decays and decays of $b$-hadrons, referred 

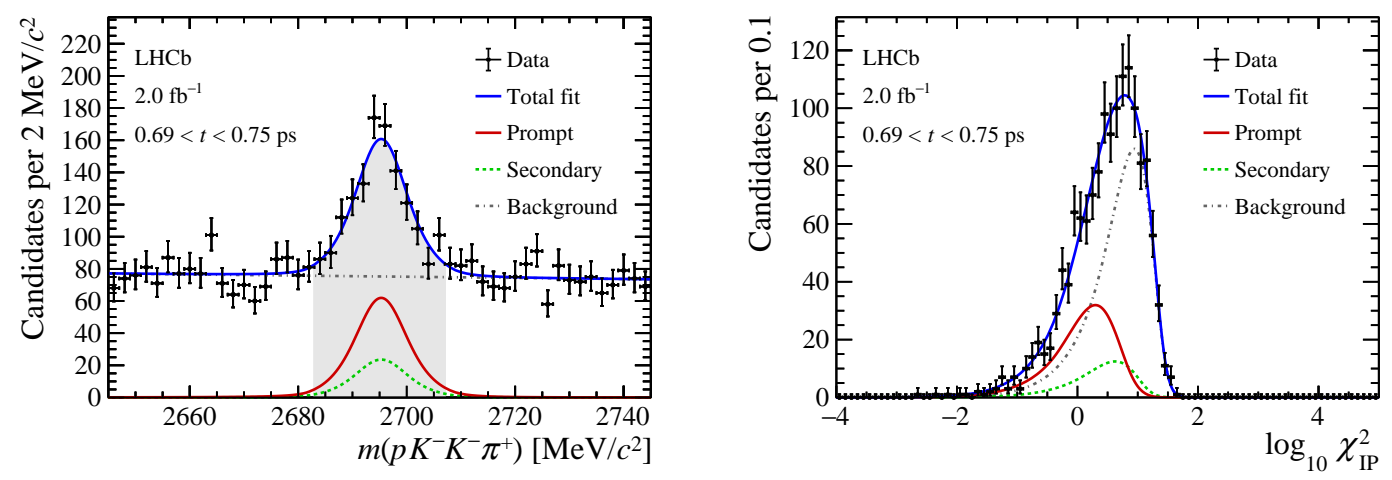

Figure 1: Distributions of the (left) invariant mass and (right) $\log _{10} \chi_{\mathrm{IP}}^{2}$ for the $\Omega_{c}^{0}$ baryon using 2018 data sample in one of the decay intervals $(0.69-0.75 \mathrm{ps})$. The red solid, green dashed, and grey dash-dotted lines represent the signal, the secondary decays, and the combinatorial background contributions, respectively.
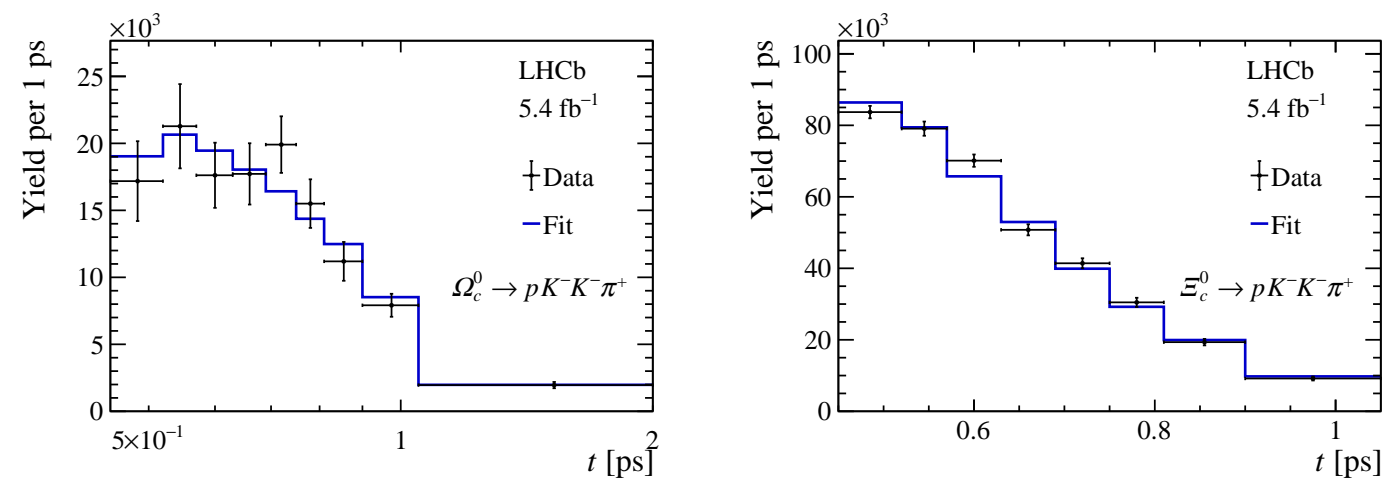

Figure 2: Decay time distribution for (left) $\Omega_{c}^{0}$ and (right) $\Xi_{c}^{0}$ with the lifetime fit represented by the blue line.

to as secondary decays. Figure 1 shows both variables for the $\Omega_{c}^{0}$ baryon in one of the decay time intervals using 2018 data sample.

\subsection{Results}

The $\Omega_{c}^{0}$ and $\Xi_{c}^{0}$ lifetimes are determined with extended maximum likelihood fits to $\left(m, \log _{10} \chi_{\mathrm{IP}}^{2}\right)$ yields simultaneously in decay time bins, which are shown in Fig. 2. The lifetimes are measured to be $\tau\left(\Omega_{c}^{0}\right)=276.5 \pm 13.4$ (stat) \pm 4.4 (syst) $\pm 0.7\left(D^{0}\right)$ fs and $\tau\left(\Xi_{c}^{0}\right)=148.0 \pm 2.3$ (stat) \pm 2.2 (syst) \pm $0.2\left(D^{0}\right)$ fs. Dominant systematic uncertainties are due to fit model, the kinematic corrections to the simulated data and decay-time resolution. It is the most precise measurement of the $\Omega_{c}^{0}$ lifetime up to date. Both $\Omega_{c}^{0}$ and $\Xi_{c}^{0}$ lifetime measurements are consistent with the previous LHCb measurement, as shown in Fig. 3. This is an additional confirmation of a newly established lifetime hierarchy $\tau\left(\Xi_{c}^{+}\right)>\tau\left(\Omega_{c}^{0}\right)>\tau\left(\Lambda_{c}^{+}\right)>\tau\left(\Xi_{c}^{0}\right)$ from the experimental side. 


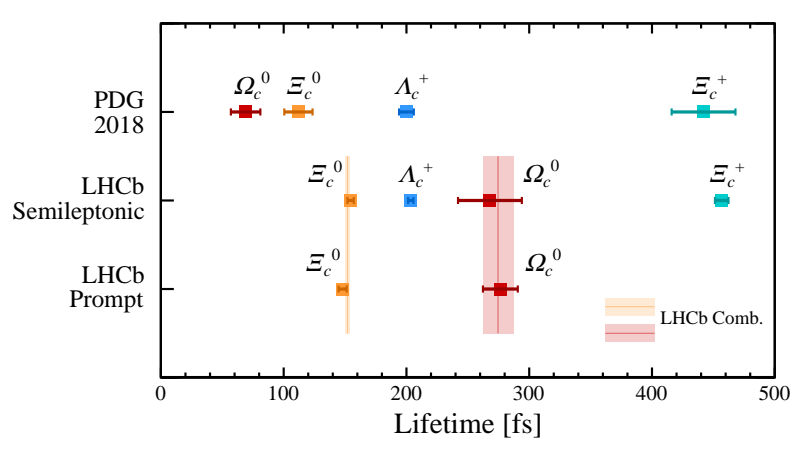

Figure 3: Results from the LHCb measurement of the $\Omega_{c}^{0}$ and $\Xi_{c}^{0}$ lifetimes using semileptonic $b$-hadron decays [3, 4] and promptly produced baryons [6], and the previous 2018 world average [5]. Coloured bands represent the combination of the $\mathrm{LHCb}$ results.

\section{Doubly charmed baryons}

\subsection{Introduction}

Doubly charmed baryon studies offer a unique platform to study the non-perturbative dynamics in the presence of two charm quarks in baryons. Moreover, a completion of the observation of all baryons in the SU(4) baryon 20-plets is of a high experimental interest. In 2017, the first observation of the doubly charmed baryon $\Xi_{c c}^{++}$(quark content $c c u$ ) in the $\Lambda_{c}^{+} K^{-} \pi^{+} \pi^{+}$final state was announced by the LHCb collaboration [7]. Further studies of the $\Xi_{c c}^{++}$baryon by the LHCb collaboration followed its observation, namely a confirmation in the $\Xi_{c c}^{++} \rightarrow \Xi_{c}^{+} \pi^{+}$decay mode [8], a search for the $\Xi_{c c}^{++} \rightarrow D^{+} p K^{-} \pi^{+}$decays [9], measurements of its lifetime [10] and production cross-section [11], and a precision mass measurement [12].

The SELEX collaboration reported an observation of the $\Xi_{c c}^{+}$baryon (quark content $c c d$ ) with a mass of $3518.7 \pm 1.7 \mathrm{MeV} / c^{2}$ in $\Xi_{c c}^{+} \rightarrow \Lambda_{c}^{+} K^{-} \pi^{+}$and $\Xi_{c c}^{+} \rightarrow p D^{+} K^{-}$decay modes [13, 14], however it was not confirmed by the subsequent searches by FOCUS [15], BaBar [16], and Belle [17]. The LHCb collaboration performed a search for the $\Xi_{c c}^{+}$baryon in the $\Lambda_{c}^{+} K^{-} \pi^{+}$final state using data sample corresponding to an integrated luminosity of $0.65 \mathrm{fb}^{-1}$ [18], with a recent update of the search for this decay using all available LHCb data corresponding to $9 \mathrm{fb}^{-1}$ of integrated luminosity [19], with no significant signal observed. Even though the production cross-section and mass of the $\Xi_{c c}^{+}$baryon are expected to be similar to its isospin partner $\Xi_{c c}^{++}$baryon, the lifetime of the $\Xi_{c c}^{+}$state is predicted to be $\sim 2-4$ times shorter than the lifetime of the $\Xi_{c c}^{++}$baryon measured to be $0.256_{-0.022}^{+0.024}$ (stat) \pm 0.014 (syst) ps [10], which makes searches for the $\Xi_{c c}^{+}$baryon more challenging.

There are no previously reported searches for the $\Omega_{c c}^{+}$baryon (quark content $c c s$ ). It is expected that its production cross-section in $p p$ collisions is about $1 / 3$ of the $\Xi_{c c}^{+}$and $\Xi_{c c}^{++}$baryons due to the presence of an $s$ quark in the $\Omega_{c c}^{+}$baryon. The mass of the $\Omega_{c c}^{+}$baryon is expected to be in the range of 3.6-3.9 GeV/ $c^{2}[20]$ and most of its lifetime predictions are in the range from 75 to $180 \mathrm{fs}$ [21]. 

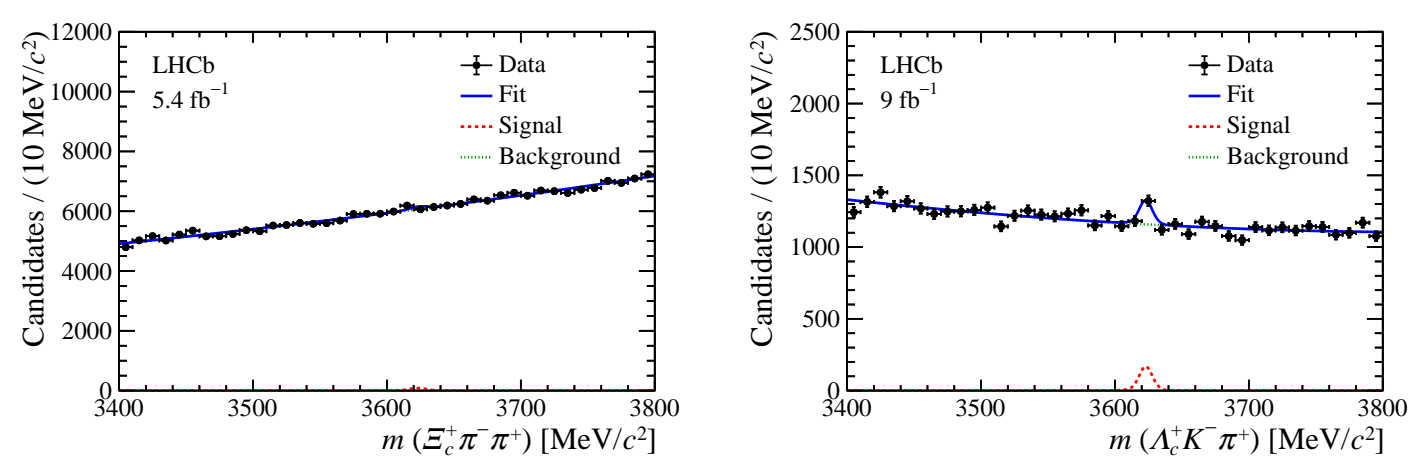

Figure 4: Invariant-mass spectra for the (left) $\Xi_{c}^{+} \pi^{-} \pi^{+}$and (right) $\Lambda_{c}^{+} K^{-} \pi^{+}$final states. The blue solid curve represents the result of a simultaneous fit to the two spectra, with the red dashed (green dotted) curve showing the signal (background) component.

\subsection{Search for the $\Xi_{c c}^{+}$baryon}

A new search for the doubly charmed baryon $\Xi_{c c}^{+}$in the $\Xi_{c c}^{+} \rightarrow\left(\Xi_{c}^{+} \rightarrow p K^{-} \pi^{+}\right) \pi^{-} \pi^{+}$decays is performed using $p p$ collisions collected by the LHCb experiment corresponding to $5.4 \mathrm{fb}^{-1}$ of integrated luminosity [22]. In order to avoid experimenter's bias, the region of the $\Xi_{c}^{+} \pi^{-} \pi^{+}$invariant mass from 3.3 to $3.8 \mathrm{GeV} / c^{2}$ was not examined until the full procedure had been finalised. Four main stages of the selection are applied to the collected data sample - a hardware and software trigger selection, a preselection based on sequential requirements, a multivariate-analysis based selection and a removal of multiple candidates. To develop the signal selection and to evaluate the ratio of efficiencies between the signal and normalisation decays, the simulated events are used as a signal proxy and an incorrect combination of final state tracks, the same-sign pions $\Xi_{c}^{+} \pi^{-} \pi^{-}$ combinations, are used as a background representation in the signal region.

The invariant mass of the selected candidates is fitted by the sum of a Gaussian function and a Crystal Ball function with power-law tails on both sides [23] with a shared mean for the signal component and an exponential function to describe the background contribution. No significant signal is found for this decay mode in the $3400-3800 \mathrm{MeV} / c^{2}$ invariant-mass window. A combined fit with the $\Lambda_{c}^{+} K^{-} \pi^{+}$final state is performed, using a sample that corresponds to the Selection B in Ref. [19]. The combined fit is shown in Fig. 4 for both $\Xi_{c c}^{+} \rightarrow \Xi_{c}^{+} \pi^{-} \pi^{+}$and $\Xi_{c c}^{+} \rightarrow \Lambda_{c}^{+} K^{-} \pi^{+}$ decays, where the fitted mass is found to be $3623.0 \pm 1.4$ (stat) $\mathrm{MeV} / c^{2}$. Figure 5 shows the local $p$-values as a function of the $\Xi_{c c}^{+}$invariant mass, without the systematic uncertainties taken into account. The minimal combined $p$-value corresponds to a local significance of $4.0 \sigma$ and a global significance of $2.9 \sigma$, with the systematic uncertainties included.

Since no significant signal is observed in the $\Xi_{c}^{+} \pi^{-} \pi^{+}$final state, an upper limit is set on the ratio of production cross-sections times the ratio of branching fractions between the signal decay and the normalisation decay $\Xi_{c c}^{++} \rightarrow \Xi_{c}^{+} \pi^{+}$, defined as

$$
R \equiv \frac{\sigma\left(\Xi_{c c}^{+}\right) \times \mathcal{B}\left(\Xi_{c c}^{+} \rightarrow \Xi_{c}^{+} \pi^{-} \pi^{+}\right)}{\sigma\left(\Xi_{c c}^{++}\right) \times \mathcal{B}\left(\Xi_{c c}^{++} \rightarrow \Xi_{c}^{+} \pi^{+}\right)}=\frac{\varepsilon_{\mathrm{norm}}}{\varepsilon_{\mathrm{sig}}} \frac{N_{\mathrm{sig}}}{N_{\mathrm{norm}}}
$$

where $\sigma\left(\Xi_{c c}^{+}\right)$and $\sigma\left(\Xi_{c c}^{++}\right)$are the production cross-sections of the $\Xi_{c c}^{+}$and $\Xi_{c c}^{++}$baryons, and $\mathcal{B}$ represents the corresponding branching fractions. The number of observed candidates for the 


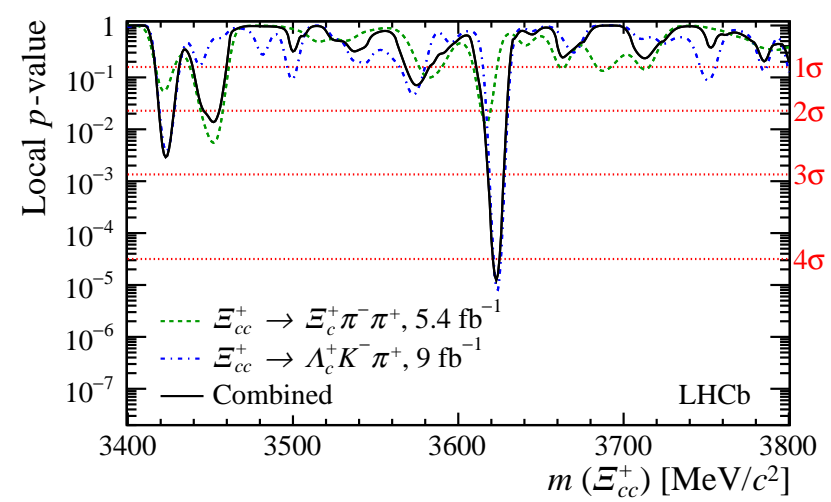

Figure 5: Local $p$-values as a function of the $\Xi_{c c}^{+}$invariant mass, for $\Xi_{c c}^{+}$baryon decays reconstructed in the $\Xi_{c}^{+} \pi^{-} \pi^{+}$(green dashed curve) and $\Lambda_{c}^{+} K^{-} \pi^{+}$(blue dash-dotted curve) modes, or combining the two modes (black solid curve). The horizontal dotted red lines represent the $p$-values corresponding to significances of $1,2,3$ and $4 \sigma$. The systematic uncertainties are not taken into account.

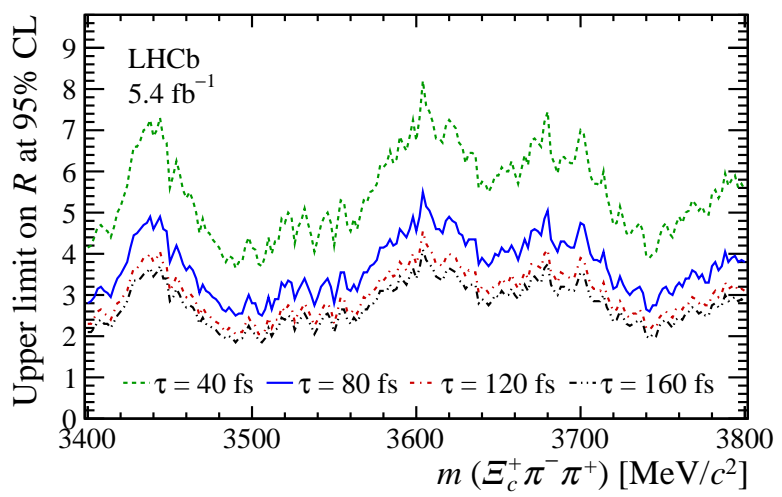

Figure 6: Upper limits on $R$ as a function of the assumed $\Xi_{c c}^{+}$mass for four different lifetime $(\tau)$ hypotheses at $95 \% \mathrm{CL}$.

signal (normalisation) mode is denoted as $N_{\text {sig }}\left(N_{\text {norm }}\right)$, and the corresponding efficiencies are $\varepsilon_{\text {sig }}$ and $\varepsilon_{\text {norm. }}$. The upper limit is evaluated as a function of mass in the invariant-mass window of $3400-3800 \mathrm{MeV} / \mathrm{c}^{2}$ for four lifetime hypotheses $-40,80,120$ and $160 \mathrm{fs}$. The CLs method [24] is used to calculate the limit at $90 \%$ confidence level (CL), which is shown in Fig. 6. The CLs curve is determined from $3 \times 10^{5}$ pseudoexperiments for each hypothetical value of $R$ and each mass with $2 \mathrm{MeV} / c^{2}$ steps in the examined invariant-mass window.

\subsection{Search for the $\Omega_{c c}^{+}$baryon}

The first search for the doubly charmed baryon $\Omega_{c c}^{+}$is performed using $p p$ collisions collected by the $\mathrm{LHCb}$ experiment corresponding to $5.4 \mathrm{fb}^{-1}$ of integrated luminosity [25]. The $\Omega_{c c}^{+}$baryon is reconstructed in the $\Xi_{c}^{+} K^{-} \pi^{+}$final state. The invariant-mass window of $3.5-4.0 \mathrm{GeV} / c^{2}$ was not examined until the full procedure had been finalised in order to avoid experimenter's bias. Two selections are developed - selection A optimised to maximise the signal sensitivity and selection B optimised to minimise the systematic uncertainties for the determination of the ratio of production 

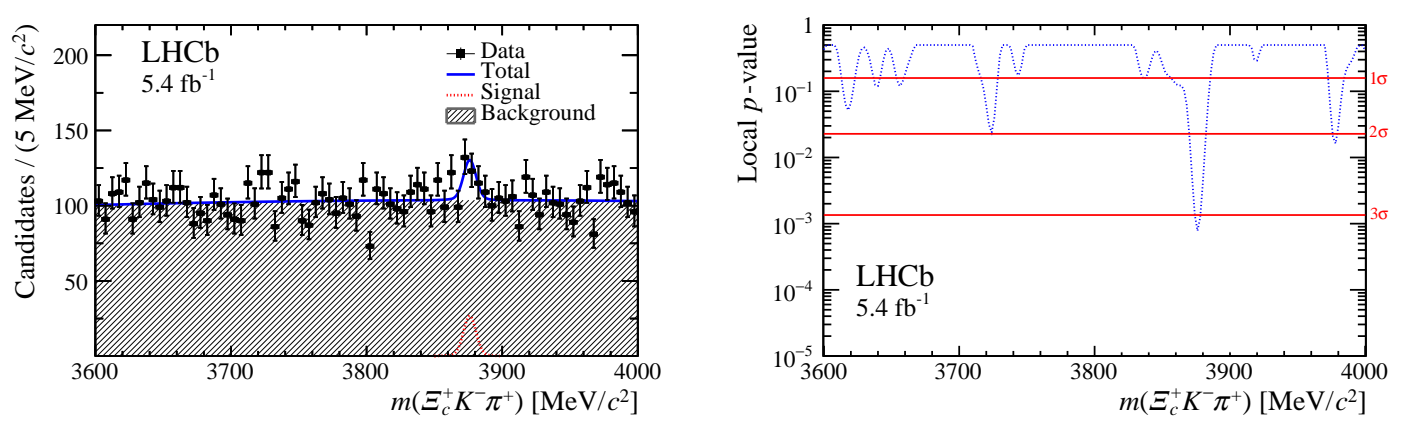

Figure 7: Distribution of the (left) invariant mass for the $\Xi_{c}^{+} K^{-} \pi^{+}$final state from selection A. The blue solid curve represents the result of the fit, with the red dotted curve (grey dashed area) showing the signal (background) component. The (right) $p$-value scan for the selection A as a function of the hypothetical $\Omega_{c c}^{+}$ mass. The horizontal red lines represent the $p$-values corresponding to significances of 1,2 and $3 \sigma$.

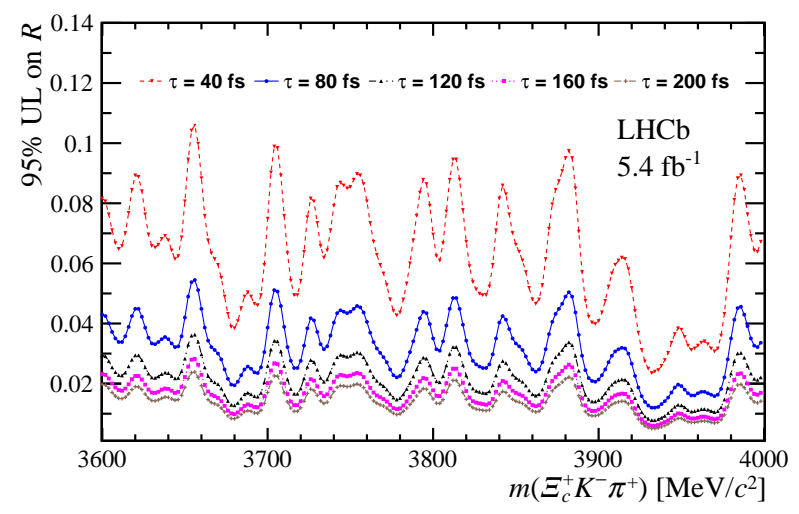

Figure 8: Upper limits on $R$ as a function of the assumed $\Omega_{c c}^{+}$mass for five different lifetime $(\tau)$ hypotheses at $95 \% \mathrm{CL}$ using selection $\mathrm{B}$.

cross-sections times the ratio of branching fractions between the signal decay and the normalisation decay $\Xi_{c c}^{++} \rightarrow \Lambda_{c}^{+} K^{-} \pi^{+} \pi^{+}$, defined as

$$
R \equiv \frac{\sigma\left(\Omega_{c c}^{+}\right) \times \mathcal{B}\left(\Omega_{c c}^{+} \rightarrow \Xi_{c}^{+} K^{-} \pi^{+}\right) \times \mathcal{B}\left(\Xi_{c}^{+} \rightarrow p K^{-} \pi^{+}\right)}{\sigma\left(\Xi_{c c}^{++}\right) \times \mathcal{B}\left(\Xi_{c c}^{++} \rightarrow \Lambda_{c}^{+} K^{-} \pi^{+} \pi^{+}\right) \times \mathcal{B}\left(\Lambda_{c}^{+} \rightarrow p K^{-} \pi^{+}\right)}
$$

The invariant mass of the selected candidates is fitted by the sum of two Crystal Ball functions [23] for the signal component and a second-order Chebyshev function is used to describe the background contribution, as shown in Fig. 7. The $p$-values evaluated as a function of the $\Omega_{c c}^{+}$ mass are shown in Fig. 7. The largest local significance is found at $3876 \mathrm{MeV} / c^{2}$, corresponding to $3.2 \sigma$. The global significance evaluated in the $3600-4000 \mathrm{MeV} / c^{2}$ mass range is estimated to be $1.8 \sigma$. All $p$-values are evaluated without the systematic uncertainties. Since no significant signal is observed, upper limits on $R$ are evaluated as a function of mass in the invariant-mass window of $3600-4000 \mathrm{MeV} / \mathrm{c}^{2}$ for five lifetime hypotheses $-40,80,120,160$ and $200 \mathrm{fs}$, as shown in Fig. 8. 

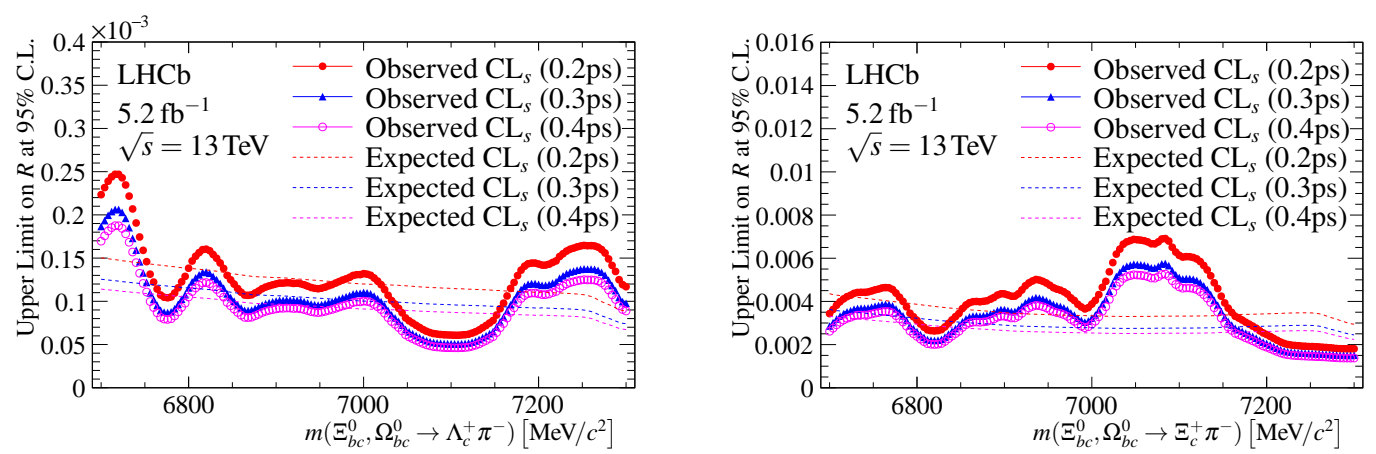

Figure 9: Upper limits on the ratio of production cross-section for the (left) $\Lambda_{c}^{+} \pi^{-}$and (right) $\Xi_{c}^{+} \pi^{-}$final states.

\section{Charm-beauty baryons searches}

\subsection{Introduction}

No baryons containing one $c$ and one $b$ quark have been observed yet, however they are expected to be produced in the $p p$ collisions at the LHC. The LHCb detector is well suited for the search of the doubly heavy baryons due to its strong tracking and particle identification capabilities, excellent secondary vertex resolution and efficient trigger system. A search for the $\Xi_{b c}^{0}$ baryon (quark content $b c d$ ) was performed for the first time by the LHCb collaboration in the $D^{0} p K^{-}$final state [26]. No significant signal was found in the invariant mass range of $6.7-7.2 \mathrm{GeV} / c^{2}$. Nevertheless, more potential decay channels are being explored to enhance a possibility of the first observation of the charm-beauty baryons at the LHCb experiment.

\subsection{Search for the $\Xi_{b c}^{0}$ and $\Omega_{b c}^{0}$ baryons}

First search for the $\Omega_{b c}^{0}$ baryon (quark content $b c s$ ) and a new search for the $\Xi_{b c}^{0}$ baryon in the $\Lambda_{c}^{+} \pi^{-}$and $\Xi_{c}^{+} \pi^{-}$final states is performed [27]. The search is performed without examination of the studied invariant-mass windows until the full procedure has been defined. No evidence for neither of these baryons is found, therefore upper limits on the ratio of production cross-sections times the ratio of branching fractions between the signal decays with respect to the $\Lambda_{b}^{0} \rightarrow \Lambda_{c}^{+} \pi^{-}$and $\Xi_{b}^{0} \rightarrow \Xi_{c}^{+} \pi^{-}$normalisation channels are set at $95 \% \mathrm{CL}$, as shown in Fig. 9. The upper limits are set in the invariant-mass window of $6.7-7.3 \mathrm{GeV} / c^{2}$ and for different lifetime hypotheses of $0.2,0.3$ and $0.4 \mathrm{ps}$, using the CLs method [24].

\section{Summary}

The latest results on the charmed baryon lifetimes and doubly-heavy baryon searches performed at the LHCb experiment are summarised. Namely, a new measurement of the lifetimes of the $\Omega_{c}^{0}$ and $\Xi_{c}^{0}$ baryons from prompt production is presented, which is in agreement with the previous $\mathrm{LHCb}$ measurement using semileptonic $b$-decays and it is the most precise measurement of the $\Omega_{c}^{0}$ lifetime up to date. The searches for the doubly charmed baryons $\Xi_{c c}^{+}$in the $\Xi_{c c}^{+} \rightarrow \Xi_{c}^{+} \pi^{-} \pi^{+}$ decay and the first search for the $\Omega_{c c}^{+}$baryon in the $\Omega_{c c}^{+} \rightarrow \Xi_{c}^{+} K^{-} \pi^{+}$decay, together with the recent 
searches for the $\Xi_{b c}^{0}$ and $\Omega_{b c}^{0}$ baryons in the $\Lambda_{c}^{+} \pi^{-}$and $\Xi_{c}^{+} \pi^{-}$final states, are described. More studies and searches of the charmed baryons are still being performed using the data recorded by the LHCb experiment in the past few years. Moreover, the upgraded LHCb detector [28] with increased instantaneous luminosity and fully software trigger system with improved efficiency will provide an unprecedented data sample for more charmed baryon studies and searches in the upcoming years.

\section{References}

[1] I.I.Y. Bigi, The QCD perspective on lifetimes of heavy flavor hadrons, hep-ph/9508408.

[2] G. Bellini, I. Bigi and P. Dornan, Lifetimes of charm and beauty hadrons, Physics Reports 289 (1997) 1.

[3] LHCв collaboration, Measurement of the $\Omega_{c}^{0}$ lifetime, Phys. Rev. Lett. 121 (2018) 092003 LHCb-PAPER-2018-028 CERN-EP-2018-175, [1807 . 02024].

[4] LHCв collaboration, Precision measurement of the $\Lambda_{c}^{+}$, $\Xi_{c}^{+}$, and $\Xi_{c}^{0}$ baryon lifetimes, Phys. Rev. D100 (2019) 032001 LHCb-PAPER-2019-008 CERN-EP-2019-122, [1906. 08350].

[5] Particle Data Group collaboration, Review of particle physics, Phys. Rev. D98 (2018) 030001.

[6] LHCB COLLABORATION collaboration, Measurement of the lifetimes of promptly produced $\Omega_{c}^{0}$ and $\Xi_{c}^{0}$ baryons, 2109.01334 LHCb-PAPER-2021-021, CERN-EP-2021-167, [2109.01334].

[7] LHCв collaboration, Observation of the doubly charmed baryon $\Xi_{c c}^{++}$, Phys. Rev. Lett. 119 (2017) 112001 LHCb-PAPER-2017-018 CERN-EP-2017-156, [1707.01621].

[8] LHCв collaboration, First observation of the doubly charmed baryon decay $\Xi_{c c}^{++} \rightarrow \Xi_{c}^{+} \pi^{+}$, Phys. Rev. Lett. 121 (2018) 162002 LHCb-PAPER-2018-026 CERN-EP-2018-172, [1807.01919].

[9] LHCв collaboration, A search for $\Xi_{c c}^{++} \rightarrow D^{+} p K^{-} \pi^{+}$decays, JHEP 10 (2019) 124 LHCb-PAPER-2019-011 CERN-EP-2019-067, [1905 . 02421].

[10] LHCв collaboration, Measurement of the lifetime of the doubly charmed baryon $\Xi_{c c}^{++}$, Phys. Rev. Lett. 121 (2018) 052002 LHCb-PAPER-2018-019 CERN-EP-2018-146, [1806. 02744].

[11] LHCв collaboration, Measurement of $\Xi_{c c}^{++}$production in pp collisions at $\sqrt{s}=13 \mathrm{TeV}$, Chin. Phys. C44 (2020) 022001 LHCb-PAPER-2019-035 CERN-EP-2019-220, [1910 . 11316].

[12] LHCв collaboration, Precision measurement of the $\Xi_{c c}^{++}$mass, JHEP 02 (2020) 049 LHCb-PAPER-2019-037 CERN-EP-2019-258, [1911 . 08594].

[13] SELEX collaboration, First observation of the doubly charmed baryon $\Xi_{c c}^{+}$, Phys. Rev. Lett. 89 (2002) 112001 [hep-ex/0208014]. 
[14] SELEX collaboration, Confirmation of the double charm baryon $\Xi_{c c}^{+}(3520)$ via its decay to $p D^{+} K^{-}$, Phys. Lett. B628 (2005) 18 [hep-ex/0406033].

[15] S.P. Ratti, New results on c-baryons and a search for cc-baryons in FOCUS, Nucl. Phys. Proc. Suppl. 115 (2003) 33.

[16] BABAR collaboration, Search for doubly charmed baryons $\Xi_{c c}^{+}$and $\Xi_{c c}^{++}$in BABAR, Phys. Rev. D74 (2006) 011103 [hep-ex/0605075].

[17] Belle collaboration, Observation of new states decaying into $\Lambda_{c}^{+} K^{-} \pi^{+}$and $\Lambda_{c}^{+} K_{\mathrm{S}}^{0} \pi^{-}$, Phys. Rev. Lett. 97 (2006) 162001 [hep-ex/0606051].

[18] LHCв collaboration, Search for the doubly charmed baryon $\Xi_{c c}^{+}$, JHEP 12 (2013) 090 LHCb-PAPER-2013-049 CERN-PH-EP-2013-181, [1310 . 2538].

[19] LHCв collaboration, Search for the doubly charmed baryon $\Xi_{c c}^{+}$, Sci. China Phys. Mech. Astron. 63 (2020) 221062 LHCb-PAPER-2019-029 CERN-EP-2019-199, [1909. 12273].

[20] D. Ebert, R.N. Faustov, V.O. Galkin and A.P. Martynenko, Mass spectra of doubly heavy baryons in the relativistic quark model, Phys. Rev. D 66 (2002) 014008 [hep-ph/0201217].

[21] H.-Y. Cheng and Y.-L. Shi, Lifetimes of Doubly Charmed Baryons, Phys. Rev. D 98 (2018) 113005 [1809. 08102].

[22] LHCв COLLABORATION collaboration, Search for the doubly charmed baryon $\Xi_{c c}^{+}$in the $\Xi_{c}^{+} \pi^{-} \pi^{+}$final state, 2109.07292 LHCb-PAPER-2021-019, CERN-EP-2021-155, [2109.07292].

[23] T. Skwarnicki, A study of the radiative cascade transitions between the Upsilon-prime and Upsilon resonances, Ph.D. thesis, Institute of Nuclear Physics, Krakow, 1986.

[24] A.L. Read, Modified frequentist analysis of search results (The CL(s) method), in Workshop on Confidence Limits, pp. 81-101, 8, 2000.

[25] LHCв collaboration, Search for the doubly charmed baryon $\Omega_{c c}^{+}, 2105.06841$ LHCb-PAPER-2021-011, [2105.06841].

[26] LHCв collaboration, Search for the doubly heavy baryon $\Xi_{b c}$ in the $D^{0} p K^{-}$final state, JHEP 11 (2020) 095 LHCb-PAPER-2020-014,CERN-EP-2020-137, [2009. 02481].

[27] LHCв collaboration, Search for the doubly heavy baryons $\Omega_{b c}^{0}$ and $\Xi_{b c}^{0}$ decaying to $\Lambda_{c}^{+} \pi^{-}$ and $\Xi_{c}^{+} \pi^{-}, 2104.04759$ LHCb-PAPER-2021-002,CERN-EP-2021-046, [2104.04759].

[28] LHCв collaboration, Framework TDR for the LHCb Upgrade: Technical Design Report, CERN-LHCC-2012-007. 